\title{
Research on Automotive Brake-by-wire System Based on FlexRay Bus
}

\author{
Xiangzhi liu ${ }^{\mathrm{a}}$, Peng Zhang ${ }^{\mathrm{b}}$, Fuqiang $\mathrm{Wang}^{\mathrm{c}}$, Yifan $\mathrm{Hu}^{\mathrm{d}}$ and Hong Liu ${ }^{\mathrm{e}}$ \\ Shandong Computer Science Center (National Supercomputer Center in Jinan) \\ Shandong Provincial Key Laboratory of Computer Networks \\ No. 19 Keyuan Road, 250014, Jinan, Shandong, P. R. China \\ $\left\{{ }^{a} l i u x z h,{ }^{b} z h a n g p,{ }^{c}\right.$ wangfq, ${ }^{d}$ huyf, liuh\}@sdas.org
}

Keywords: FlexRay; brake-by-wire; electronic control unit

\begin{abstract}
The vehicle's next generation presents new requirements for high performance, high reliability and high stability of the body layout and electronic control unit (ECU). Using FlexRay bus to realize the ECU is an ideal solution for those requirements. The FlexRay technology can effectively improve the vehicle's safety and stability, while eliminating the traditional mechanical or hydraulic control devices. This paper has researched the vehicle brake-by-wire (BBW) system, which bases on FlexRay bus. The BBW is mainly composed of the motor driver module, the main control board, and the action board. The BBW system prototype has been constructed, and the vehicle's feasibility and practicability are verified on site. The results show that the system performances meet the design requirements.
\end{abstract}

\section{Introduction}

Nowadays, automotive electronic technology is developing rapidly in the direction of intelligence, modularization and network. Automotive electronics technology is the main driving force for the vehicle's industry development in the world. It is the main technical ways to improve the safety, reliability, comfort, environmental protection, and energy saving. With the degree of automotive electronics increasing, the number of electronic control unit (ECU) modules is also increases, so that the wire harness increase sharply also. Because of the strict control of the manufacturing cost and the automotive body weight, reducing the wire harness weight has becomes a problem that must be solved. In the braking system specifically, the involving ECU may turn into the $\mathrm{x}$-by-wire ( $\mathrm{x}$ is stands for steering, braking, throttle, clutch, etc.) in the future [1]. Since the BBW system is a distributed real-time system involving safety, it will also have higher requirements on the bus technology [2]. The bus technology has widely used in the vehicle, such as controller area network (CAN), vehicle area network (VAN), local interconnect network (LIN), etc. For lacking the certainty, synchronization, and fault tolerant mechanism, those current buses cannot meet the requirements of the x-by-wire control.

Proposed by FlexRay ${ }^{\mathrm{TM}}$ consortium, FlexRay is the ideal solution for automotive x-by-wire system. FlexRay ${ }^{\mathrm{TM}}$ consortium consists of vehicle manufacturers and MCU makers, such as Bayerische Motoren Werke (BMW), BOSCH, General Motors (GM), VolksWagen (VW), Daimler, Chrysler, NXP, Motorola, and Philips. As one of the most advanced vehicle bus technologies, FlexRay is a hot spot in the research of vehicle network [3-5]. Because of its high performance, FlexRay meets the requirements of the future vehicle. Compared with the CAN bus, which has been widely used in vehicle network, FlexRay has the following characteristics [6].

1) Higher transmission rate. FlexRay supports two communication channels. The data transmission of each channel reaches $10 \mathrm{Mbits} / \mathrm{s}$ (Mbps). Compared with the CAN bus, it can increase the available bandwidth by 10-40 times (depending on the configuration and comparison mode), and supports more than $90 \%$ bus utilization.

2) Synchronous time base. The time base is automatic established, synchronized, then it is provided to application system. The accuracy is between 0.5 and $10 \mu \mathrm{s}$ (usually $1 \sim 2 \mu \mathrm{s}$ ). 
3) Time triggered mechanism. Communication is carried out in a cycle. The specific message has a fixed position in the communication cycle, so the receiver knows the message's arrival time in advance. The temporary deviation of arrival time is very small, so the arrival time can be guaranteed.

4) Redundant communication. Support for dual channel redundancy, and have perfect fault tolerance algorithm.

5) Flexible. Message can be redundant or non-redundant transmission. Bandwidth can be allocated statically or dynamically. Extending system does not need to adjust the software in the existing node. A variety of topologies, such as bus, star and hybrid, are supported.

X-by-wire technique originated from aircraft control system. It changes the pilot control commands into electrical signals $[7,8]$. The vehicle's control system can be based on x-by-wire technology. The x-by-wire technology changed the traditional automotive mechanical system into electrical system, which is realized by the central processing unit (CPU) of high performance and the communication bus of high speed fault tolerant. X-by-wire technology will reduce the vehicle weight, fuel consumption, and complexity of automotive electronic components. Through this technology, the mechanical system is replaced by the electronic system. At the same time, $x$-by-wire system will become more simple and efficient in many respects, such as manufacturing, assembly, and testing. It can be designed and realized using modular technology also. The x-by-wire technology will help the technicians to improve the free space of the vehicle design, reduce the manufacturing cost, and improve the vehicle's stability and safety.

\section{Construct BBW System}

\section{A. BBW System Components}

The designed BBW system is mainly composed of three parts, the experiment board based on the MC9S12XF512 series MCU, motor driver module, and the power supply. The vehicle electronic control system unit is used the typical design $[9,10]$. The electronic control system unit is connected to the FlexRay node through the FlexRay communication module. Figure 1 shows the schematic structure of BBW prototype system.

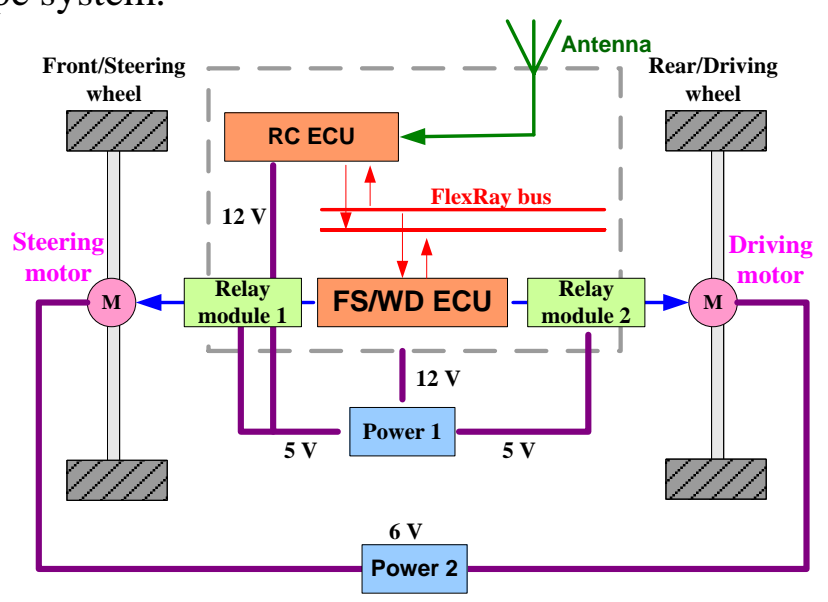

Fig. 1 Brake-by-wire prototype system.

Abbreviations: RC: Remote Control; FS/WD: Front-wheel Steer/Wheel Drive; V: volt.

The experiment board includes the main control board and the action board. The main control board receives the control signal. The action board adopts bidirectional FlexRay communication with the main control board, receives control instruction, and then does the corresponding control actions.

The motor drive module adopts a double relay control motor, and it is connected with the action board to control the steering motor and the driving wheel motor.

In this BBW system, the motor and the experiment board adopt independent power supply isolation scheme. The experiment board uses $12 \mathrm{~V}$ battery to supply power directly, and relay module is supplied by $5 \mathrm{~V}$ direct current (DC) power module. Powered by $6 \mathrm{~V}$ battery, the motor drive module controls driving and steering motor by DC. 


\section{B. Realization of BBW}

1) Signal shape

Definite signal forms, such as forward, backward, stop drive, turn left, turn right, stop turning.

Connected to the $2.4 \mathrm{GHz}$ remote control module output interface, the FlexRay experimental board is based on the MC9S12XF512 MCU. The MCU is provided with a pulse interrupt timer, and the output signal of the remote control module is collected by the interrupt program. The program is mainly to achieve those following functions.

- $\quad$ To judge interrupt trigger signal status.

- To eliminate the interference signal.

- To analysis signal channel and signal shape, determine remote control action.

- $\quad$ To get FlexRay command code.

2) FlexRay communication processing

Configure FlexRay communication mode. The NXP ${ }^{\circledR}$ 16-bit MC9S12XF512 MCU is composed of an XGATE coprocessor, 512 KB flash memory and an embedded FlexRay controller. XGATE kernel is responsible for handling FlexRay communications. Once the interrupt happening, control signal is treated by the kernel module. The command code is stored in the FlexRay data transmission buffer unit, which triggers the XGATE to send data interrupt, so the command code is sent through the FlexRay interface.

In this paper, the main control board sends the FlexRay command code to the action board, and it synchronizes FlexRay communication too. The action board receives the FlexRay command code, which is directly analysis by the FlexRay interrupt handler. The XGATE kernels outputs the control signals to the related port, and then control the wheel drive motor and steering motor work.

Brake mode. Brake the motor by short circuit.

\section{The Main Control Board and the Action Board}

Based on the NXP ${ }^{\circledR}$ 16-bit MC9S12XF512 MCU, both boards have the following features.

- $5 \mathrm{~V}$ regulator circuit, input DC 5-24 V.

- $\quad$ All functional pins through the interface.

- 1 CAN bus, using PCA82c250/TJA1040 chip and HCPL2630 photoelectric coupling chip.

- 1 LIN interface.

- 1 RS232 serial port interface.

- 2 FlexRay bus, using TJA1080ATS transceiver.

D. Motor Drive Modes

The output of the double relay interface forms three status: forward, backward, and braking. Table 1 shows the motor driver modes.

Table 1 Motor driver modes

\begin{tabular}{|l|l|c|l|l|}
\hline Relay A & Relay B & Motor driver voltage (V) & Motor status & Vehicle status \\
\hline power on & power off & +6 & corotation & forward \\
\hline power off & power on & -6 & reverse & backward \\
\hline power on & power on & 0 & Short circuit, stop & braking \\
\hline power off & power off & 0 & Short circuit, stop & braking \\
\hline
\end{tabular}

\section{Experimental Results}

From the experimental results, the data transmission time interval can be further reduced, and the minimum time gap of sending data is about $0.5 \mu \mathrm{s}$. When the time gap reaches $0.5 \mu \mathrm{s}$, the FlexRay transmission rate will achieve about $10 \mathrm{Mbps}$, which meet the high speed communication needs of vehicle data network in the next generation.

\section{Analysis and Discuss}

At any time, the BBW control system must collect enough information to guarantee precision control the vehicle. More and more sensors are used in vehicles, especially those sensors which are used to 
sense the road surface information. Such sensors include video, radar, and optical sensors. A large amount of sensing data is transmitted to the ECU in real time. In order to realize the actual operation of BBW system, it is necessary to solve many design's problems, such as data rate, network topology, node synchronization, and node structure.

\section{A. Design Data Rate and Network Topology}

The BBW system designs two independent physical circuits for FlexRay communication. The data rate for each circuit is up to $10 \mathrm{Mbps}$. The two communication circuits are mainly used to achieve redundancy, and the message transmission has the ability of fault tolerance.

The design takes into account that the FlexRay can also operate at low data rate. Once the data rate is less than $1 \mathrm{Mbps}$, it is allowed to support other bus structure, e.g., CAN bus.

\section{B. FlexRay Nodes Synchronization}

Every FlexRay node includes a controller and a driver. The controller includes a host and a communication controller. Driver usually includes bus driver and bus guardian (optional). The bus driver connects the communication controller with the bus. The host notifies bus guardian that the communication controller has been assigned what time-slots. The bus guardian only allows the communication controller to transmit data in these time-slots, and activates the bus driver. If the bus guardian finds a time interval, the connection of the communication channel is disconnected.

The FlexRay node has been designed several following basic operating statuses:

Configuration status (default): For setting a variety of initialization parameters, including communication cycles and data rates.

Ready status: For setting internal communication parameters.

Wake up status: For waking up nodes which are not in the communication status. In this status, the node sends a wake-up signal to another node for wakening up and activating the bus driver, communication controller, and bus guardian.

Startup status: For starting clock synchronization and preparing for communication.

Normal status (active/passive): The status of being able to communicate.

Interrupt status: Indicates communication has been interrupted.

The FlexRay node has a state transition which associated with error handling. When the individual node's clock is different from the FlexRay synchronization node's clock, the clock correction error occurs. The FlexRay network has one or more synchronous nodes that transmit synchronization information. After receiving any synchronization information, the node compares its clock with the synchronization node's clock, and makes the necessary changes according to the synchronization information.

Every node must count errors. At the same time, the node also tracks the errors associated with the frame transfer/accept status, including syntax errors, content errors, bus interference errors, and the errors caused by the transfer conflicts. Once a node has found the error, it will notify the host.

In order to realize the synchronization function, and optimize the bandwidth by using the short distance between two pieces of message, those components of the communication network must have a common time base, i.e., global time. Synchronous information is transmitted in the static state of the cycle. For synchronization, all local clocks are corrected with the global clock.

\section{FlexRay Network Node Structure}

The FlexRay network node is composed of a main processor, a FlexRay communication controller, a bus guardian (optional), and a bus driver. The main processor provides and generates data, and transmits it through the FlexRay controller. The bus driver is connected with the communication controller and the bus, or it is connected with the bus guardian and the bus. The main processor notifies the bus guardian of the time-slots allocated by the FlexRay controller, and the bus guardian allows the FlexRay controller to transmit data in these time-slots.

The FlexRay communication frame has three frame types: the head segment, the load segment, and the tail segment. FlexRay uses a time triggered protocol to transfer frame types. In according with the predetermined schedule, FlexRay time trigger mode ensures that the data can be transmitted.

- The header segment includes the following: 
Reserved bit - Prepare for future extensions.

Payload preamble indicator bit - In a static frame and dynamic frame, this bit specifies the Network Manage Vector and the message ID, respectively.

Null frame indicator bit - Indicates the data frame of the load segment is null or not.

Sync frame indicator bit - Indicates a synchronous frame.

Startup frame indicator bit - Indicates that the frame is the startup frame or not.

Frame ID (11 bits) - The ID allocated to each node in the system design process (range: 1-2047).

Payload length (7 bits) - The payload segment data length.

Header CRC (11 bits) - The calculated CRC value.

Cycle count (6 bits) - The cycle number of nodes that transmit frames.

- The payload segment:

Message Data - 0-254 bytes. First two bytes can be used for message ID.

- The trailer segment

The trailer segment includes the CRC value specified by the hardware. These CRC values change the seed value in the connected channel to prevent incorrect correction.

\section{Summary}

This paper designs a BBW prototype system. This system is verified on-site, and results show that it satisfies the design requirements. Of course, the x-by-wire system is not only limited to the braking system. It can be applied to other vehicle's control system, such as the clutch system, the steering system, the transmission system, and the suspension system.

\section{Acknowledgements}

This work was supported in part by the National Nature Science Foundation of China (No. 61401257, 61501282, 61601267), Shandong Academy Young Scientists Fund Project (No. 2013QN037), Shandong Academy Basic Research Fund, Shandong Academy of Sciences Pilot Project for Science and Technology.

\section{References}

[1] E. A. Bretz: IEEE Spectr. Vol. 38, no. 4 (2001), p. 68.

[2] R. Isermann, R. Schwarz, and S. Stolzl: IEEE. Control Syst. Mag. Vol. 22, no. 5(2002), p. 64.

[3] Z. H. Gu, G. Han, H. B. Zeng, and Q. L. Zhao: IEEE Trans. Parallel Distrib. Syst. Vol. 27, no. 10(2016), p. 3044.

[4] Y. X. Chen and Z. G. Liu: App. Mech. Mat. Vol. 327(2013), p. 1019.

[5] C. Muller, M. Valle, R. Buzas, and Filip Brtan: J. Circuit Syst. Comp. Vol. 25, no. 11(2016), article number: 1650137.

[6] Information on http://www.FlexRay.com.

[7] T. Dan: 45th Annual Frontiers in Education Conference, Camino Real El Paso, El Paso, TX, USA, 2015, p.1.

[8] D. Li, Y. X. Chen, H. C. Xiang, and F. Son: Tele. Eng. Vol. 54(2014), p. 1700.

[9] D. Waraus: Appl. Elec., p. 269, IEEE(2009).

[10]I. Park and M. Sunwoo: IEEE Trans. Ind. Electron. Vol. 58(2011), p. 1449. 